\title{
Mediación pedagógica de lecturas en inglés para el estudiantado con un nivel básico del idioma en la Cátedra de Educación Preescolar en la UNED, Costa Rica
}

\author{
English reading pedagogical mediation for language basic level students in \\ the Preschool Education course at UNED, Costa Rica
}

\section{Mediação pedagógica de leituras em inglês para estudantes com um nível . básico da língua.no. curso de Educação Pré-Escolar da UNED, Costa Rica}

\author{
Delia María Solís Solís \\ Universidad Estatal a Distancia \\ San José, Costa Rica \\ dsolis@uned.ac.cr \\ (1) ORCID: https://orcid.org/0000-0002-8844-2035 \\ Katherine Acevedo Nevermann \\ Universidad Estatal a Distancia \\ San José, Costa Rica \\ kacevedo@uned.ac.cr \\ (1) ORCID: https://orcid.org/0000-0003-3128-0642
}

Recibido - Received - Recebido: 15 / 09 / 2021 Corregido - Revised - Revisado: 29 / 10 / 2021 Aceptado - Accepted - Aprovado: 17 / 11 / 2021

DOI: https://doi.org/10.22458/ie.v24i36.3855

URL: https://revistas.uned.ac.cr/index.php/innovaciones/article/view/3855

\begin{abstract}
Resumen: En Costa Rica, la necesidad de desarrollar competencias lingüísticas en un segundo idioma durante la primera infancia ha sido causa de que las universidades estatales inicien un proceso de revisión de sus planes de estudio para la formación de educadores. Esta realidad y la mediación de materiales en inglés, como requisito de acreditación, exigen la incorporación de lecturas en un segundo idioma en los programas de estudio de las asignaturas de la Carrera de Educación Preescolar de la UNED. Este estudio está destinado a mediar lecturas en inglés basándose en tareas y trabajo cooperativo, en estudiantes de diplomado con un manejo básico del idioma; estas estrategias resultan apropiadas para el trabajo a distancia. El material elegido por expertos fue una lectura y un video, los cuales cumplen los siguientes requisitos: 1) la lectura: debe ser corta (no más de 5 páginas) con un vocabulario simple e imágenes ilustrativas; 2) el video: corto (no más de 4 minutos) y con subtítulos en inglés. La mediación y evaluación del material se realizó en la plataforma, a través de actividades de predicción. Las actividades evaluaron la habilidad de escucha y comprensión, y se presentaron en un foro académico. Un hallazgo muestra que mientras la población estudiantil pueda utilizar todos los recursos visuales, auditivos y conocimientos previos en las actividades de evaluación, el idioma como tal no representa un obstáculo. Los resultados muestran que el beneficio de la estrategia del trabajo en tareas, el trabajo colaborativo y las actividades de predicción son técnicas adecuadas para mediar el material en otro idioma.
\end{abstract}

Palabras claves: Estrategias de aprendizaje - actitudes lingüísticas - lenguaje expresivo -habilidades lingüísticas - aprendizaje de un segundo idioma

\begin{abstract}
The need to develop linguistic competencies in a second language in Costa Rica's early childhood has led state universities to review their curricula for teacher training. This reality and the mediation of materials in English as a requirement for accreditation led to the need to incorporate readings in a second language in the different subjects of the Early Childhood Education Program at UNED, knowing that the population lacks proficiency in this language. The intention of this study was to mediate readings in English using strategies based on tasks and cooperative work for graduate students with a basic command of the language. Both strategies were chosen because they were appropriate for distance learning. The material chosen by experts was a reading and a video, which meet the following requirements: 1- the reading: short (no more than 5 pages),
\end{abstract}


simple vocabulary, and illustrative images. 2- the video: short (no more than 4 minutes) and with English subtitles. The mediation and evaluation of the material were carried out on the platform through prediction activities. The activities evaluated comprehension and listening skills and were presented in an academic forum. It was found that as long as the student population can use all visual, auditory, and prior knowledge resources in the assessment activities, language as such does not represent an obstacle. These results suggest the benefit of the strategy of task work, collaborative work, and predictive activities as techniques to mediate the material in another language.

Keywords: Learning strategies, linguistic attitudes, expressive language, language skills, second language learning

Resumo: A necessidade de desenvolver competências linguísticas em uma segunda língua na educação infantil na Costa Rica levou as universidades estaduais a rever os seus currículos de formação de formadores. Esta realidade e a mediação de materiais em inglês como requisito para a acreditação levou à necessidade de incorporar leituras em uma segunda língua nas diferentes disciplinas do curso de Educação Infantil na UNED, sabendo que a população carece de proficiência nesta língua. A intenção deste estudo era mediar leituras em inglês com a estratégia baseada em tarefas e trabalho cooperativo em estudantes de graduação com um domínio básico da língua. Ambas as estratégias foram escolhidas como adequadas para o trabalho à distância. O material escolhido pelos especialistas consistiu em uma leitura e um vídeo, que cumprem os seguintes requisitos: 1- uma leitura: curta (não mais de 5 páginas), vocabulário simples e imagens ilustrativas. 2- um vídeo: curto (não mais de 4 minutos) e com legendas em inglês. A mediação e avaliação do material foram realizadas na plataforma, através de atividades de previsão. As atividades avaliaram as capacidades de compreensão e de escuta e foram apresentadas em um fórum acadêmico. Verificou-se que, desde que a população estudantil possa utilizar todos os recursos visuais, auditivos e de conhecimento prévio nas atividades de avaliação, a língua enquanto tal não representa um obstáculo. Estes resultados sugerem o benefício da estratégia de trabalho por tarefas, trabalho colaborativo e atividades de previsão como técnicas de mediação do material em língua estrangeira.

Palavras-chave: Estratégias de aprendizagem, atitudes linguísticas, linguagem expressiva, aptidões linguísticas, aprendizagem de segundas línguas.

\section{INTRODUCCIÓN}

Esta investigación surge de la preocupación de mediar material didáctico en un segundo idioma (en este caso lecturas en inglés) destinado a una población estudiantil cuyo conocimiento de la lengua es apenas básico. De dicho interés surgieron las siguientes interrogantes: ¿cuáles estrategias de mediación psicopedagógica requiere el estudiantado para atender material didáctico, en este caso lecturas en inglés, sin contar con un manejo óptimo de la lengua?, ¿es el nivel del idioma de los materiales seleccionados un aspecto fundamental por considerar?, ¿cuál periodo (al inicio, a la mitad o al final del cuatrimestre) resulta el momento propicio para realizar las actividades? El material didáctico no persigue que la población estudiantil aprenda a hablar inglés, sino que se familiarice con lecturas que complementen su aprendizaje en otro idioma, y así enriquezca su quehacer docente.

La realidad de la población costarricense, en cuanto al dominio de un segundo idioma, es clave en el proceso de familiarización con el material didáctico (en este caso lecturas en inglés) por parte de la población estudiantil. Por ejemplo, en el 2014, el ranking mundial del dominio del inglés EF EPI, ubicó a Costa Rica en el puesto 43 de 63 naciones, un nivel muy bajo. Unimer, mediante el estudio Gentico, confirma esta información; solo 450 de los 1.802 entrevistados, entre 12 y 80 años, dijeron hablar inglés (al menos en un nivel básico) y 1.351 reportaron no conocer la lengua. (Cerdas, 2014)

Cinco años después, English Proficiency Index 2019, de Education First revela que Costa Rica presenta el segundo mejor inglés no nativo de Latinoamérica (EF-EPI, 2019). El país lidera 16 naciones cuyo nivel de inglés es moderado, siendo el trigésimo a escala global (Garza, 2019). Esto contrasta con la posición de Costa Rica según el ranking mundial del 2014. Sin embargo, cabe preguntarse: ¿cómo se obtuvieron estos nuevos resultados, según los cuales Costa Rica se posiciona en un segundo lugar? A este dato se llegó mediante la aplicación de una prueba aplicada en línea a 2,3 millones de voluntarios. San José se coloca entre las ciudades con mejor dominio del inglés y suma 59,3, según la escala utilizada (Garza, 2019). 
Figura 1. Países con mejor inglés no nativo en América Latina (puntuaciones)

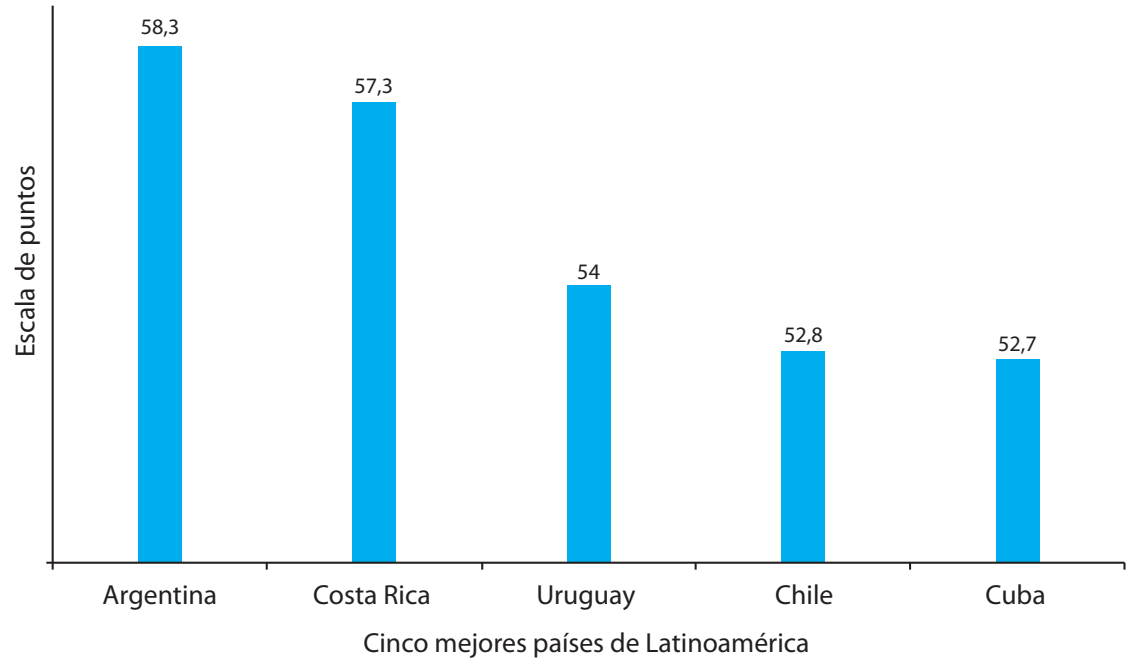

Nota: Esta gráfica muestra las puntuaciones de los países con mejor inglés no nativo en América Latina. Costa Rica presenta la segunda mejor posición en el manejo de la lengua en Latinoamérica.

Fuente: Elaboración propia con base en Garza (2019).

Así las cosas, según los estudios mencionados en el 2014 solo un $25 \%$ de la población costarricense mostró un moderado manejo del inglés. No obstante, aunque en el 2019 se evidencia una mejora en el tema, se está de frente a otra problemática fundamental: la mayoría de los docentes de inglés en Costa Rica carece de las estrategias pedagógicas indispensables para mejorar el rendimiento y la motivación de los estudiantes en las aulas (Barquero, 2019).

De acuerdo con el Diálogo Interamericano y la Editorial Pearson (2019, citado en Barquero, 2019), Costa Rica cumple con un $30 \%$ de nivel C1 en los docentes de inglés, un $51 \%$ de ellos se halla en un nivel intermedio, mientras que más de uno de cada diez docentes de inglés posee un nivel básico. Estas circunstancias influyen directamente en la mediación de los materiales, ya que el entorno es fundamental en la producción del aprendizaje.

Estos indicadores son un signo de alarma para el país, pues el dominio de un segundo idioma constituye un requisito indispensable para aspirar a mejores puestos de trabajo. Por lo tanto, el tema debe situarse a la cabeza en la lista de prioridades de los programas de formación universitaria. Al respecto, García (2016) indica: "(...) en un mundo cada vez más globalizado, es necesario aprender otro idioma aparte del materno" (p. 4).

El aprendizaje es un proceso auto organizativo y constante, el cual consiste en una transformación a partir de las experiencias de la vida, incluyendo la percepción y las emociones; además, el proceso es personal y colectivo al mismo tiempo e implica una adaptación al mundo en el cual se vive; pretende descubrir lo nuevo; se trata de una reinvención dada a partir de desaprender lo aprendido para volver a aprender.

En consecuencia, el cuerpo del ser humano está organizado según un ciclo de acoplamiento sensorio-motriz, Varela (1997). Todo cuanto se hace se realiza dentro de un ciclo de percepción-acción; por tanto, se aprende a través de todos los sentidos, ya que el cuerpo está compuesto de terminaciones nerviosas que facilitan la construcción del aprendizaje por medio del olor, la visión, el tacto, la emoción, el cariño, el lenguaje y la experiencia. Se cree que el cerebro requiere del abrazo para aprender (Assmann, 2002), pues se trata de un proceso relacionado con el placer y el disfrute tanto como con el cuerpo. 
Por lo tanto, los seres humanos deben comprender que son un compendio de experiencias formales y vivenciales, surgidas de procesos espontáneos y emergentes, sin propósitos intencionales o consiente. (Assmann, 2002)

Así, el aprendizaje toma lugar en todo momento, pues la adquisición de conocimiento se da como producto de un proceso emergente y constante de los sistemas vivos en interacción con el medio. Se trata de una acción continua propia de la vida. Ahora bien, en tanto todos los seres vivos aprenden, es necesario considerar que el aprendizaje se potencia cuando es significativo para el aprendiente y se relaciona con la experiencia del placer.

En términos generales la vida está conformada por una red autoorganizada, gracias a la plasticidad y flexibilidad interna de los sistemas y sus relaciones dinámicas, mediante la cual se produce el cambio y la capacidad para adaptarse al cambio. Este estudio consideró mediar lecturas en inglés con estrategias pedagógicas favorecedoras del aprendizaje individual y grupal.

Para un óptimo aprovechamiento del proceso de enseñanza-aprendizaje, las personas estudiantes deben estar motivadas, por lo cual la mediación psicopedagógica adquiere un carácter prioritario, razón por la cual este estudio no se concentra específicamente en el aprendizaje de un segundo idioma (inglés), sino en mediar el material necesario para tal efecto. No debe existir un ambiente de angustia y hostilidad hacia el idioma; de hecho, lo ideal es propiciar en todo momento un ambiente de curiosidad y un amigable acercamiento al conocimiento. Rodríguez (2014) puntualiza en este sentido: "las situaciones en la clase de idiomas son muy variadas e informales, pues a menudo los docentes incorporan actividades que, en ocasiones, comprometen emocionalmente a los participantes y que generan sentimientos de pérdida de control, incompetencia o pasión" (p. 182).

Para el aprendizaje de un segundo idioma se recomienda la aplicación de las estrategias metodológicas: la basada en tareas y el aprendizaje cooperativo, cuya aplicación es ideal para ambientes no presenciales. La estrategia basada en tareas es fundamental, ya que la lengua nunca es objeto de estudio sino que es una herramienta de la vida real que permite realizar tareas relacionadas con los conocimientos previos de cada estudiante. Es decir, el conocimiento del estudiante define la tarea (Richards y Rodgers, 2001).

El aprendizaje corporativo se centra en desarrollar actividades cooperativas planificadas y altamente interactivas centradas en el estudiante. Cada estudiante es responsable de su propio aprendizaje y es motivado a propiciar el aprendizaje de otros mediante el intercambio, socialmente estructurado, de información entre pares de estudiantes y grupos pequeños (McGroarty, 1993; Olsen y Kagan, 1992).

González y Garbanzo (2016) afirman que el aprendizaje cooperativo, al trabajarse con grupos pequeños, beneficia a la persona discente en su forma de aprender, ya que se comparten experiencias y materiales, y se maximiza la asimilación de conocimientos de los integrantes del grupo mediante el apoyo mutuo y el establecimiento de metas comunes.

Siguiendo la misma línea, se plantean actividades con ítems de predicción. La Agencia Colombiana de la Calidad de la Educación (2019) plantea que, en las actividades con predicciones, la población estudiantil debe utilizar los conocimientos previos, la información disponible en su memoria y su contexto, las ilustraciones, el título y cualquier otra información que le facilite responder y predecir las oraciones planteadas en la actividad.

En este sentido el falso y verdadero se consideró como un ítem de predicción óptimo para trabajar las lecturas en inglés con cada estudiante, pues mediante esta actividad los discentes no requieren un dominio alto del idioma para completar las preguntas. Con este estudio, se busca sustentar las bases teóricas existentes y aportar mediante una guía metodológica la mediación de material didáctico en inglés con los estudiantes que poseen un dominio básico del idioma. Las técnicas de revisión estarán amparadas 
en metodologías y esquemas internacionales para establecer y emitir conclusiones y recomendaciones con base en las técnicas de investigación utilizadas.

Por consiguiente, los objetivos de esta investigación se centraron en: 1- analizar las estrategias pedagógicas para mediar el material didáctico en inglés con estudiantes cuyo dominio del idioma es básico, para cuyo logro fue necesario determinar el grado del manejo del inglés por parte del estudiantado; 2- describir las características de fondo y forma de los materiales didácticos a utilizar en la mediación pedagógica; 3- definir el periodo académico indicado dentro del cuatrimestre para aplicar las actividades de mediación. Como producto de este trabajo se pretende proveer de herramientas técnicas a los profesionales de la enseñanza del inglés para facilitarles los procesos de revisión, mediación y evaluación del inglés.

\section{MATERIALES Y MÉTODOS}

En este estudio se aplicó una metodología mixta, puesto que se trata de "(...) un proceso que recolecta, analiza y vierte datos cuantitativos y cualitativos, en un mismo estudio" (Tashakkori y Teddlie, 2003, citado en Barrantes, 2014, p. 100).

Esta investigación sigue la propuesta del Scholarship of Teaching and Learning (SoTL), pues implica las siguientes cuatro funciones:

- Formular preguntas significativas sobre el aprendizaje de los alumnos y sobre las actividades de enseñanza diseñadas para facilitar el aprendizaje de los alumnos.

- Responder a esas preguntas haciendo visible, en primer lugar, el aprendizaje relevante de los alumnos como evidencia del pensamiento y del aprendizaje mismo (buen o mal aprendizaje), y analizando después sistemáticamente esta evidencia.

- Publicar los resultados de dicho análisis para invitar a la revisión y contribuir al conjunto de conocimientos acerca del aprendizaje de los alumnos en diversos contextos.

- Mejorar el aprendizaje de los alumnos reforzando la práctica de la enseñanza (la propia y la de los demás) [traducción propia] (Chick, s.f., párr. 2)

Mediante esta propuesta es posible sistematizar las estrategias de integración en el quehacer docente, con el fin de generar sinergia e interconexión entre las prácticas profesionales de docencia y de investigación, a partir del diseño de instrucciones y de programas de desarrollo profesional (Chocarro, Sobrino \& González, 2013).

Asimismo, la investigación se lleva a cabo en los procesos de gestión de la formación realizada por cada tutora. El procedimiento general implicó el trabajo en dos habilidades lingüísticas, a saber, la escucha y la comprensión por parte la persona estudiante a nivel de diplomado en la Carrera de Educación Preescolar de la UNED, mediante el desarrollo de una actividad con preguntas de falso y verdadero, desarrollada por cada estudiante que respondió en la plataforma.

La investigación se realizó en tres asignaturas de este nivel con una población general de 653 estudiantes. La mediación pedagógica, componente fundamental en este estudio, se realizó desde el aula virtual de cada asignatura. Con el propósito de acabar con las limitaciones en la adquisición del idioma inglés e incentivar a la persona estudiante para que esté dispuesta a participar en la aventura, se elaboró primero una actividad de diagnóstico, con el fin de conocer el grado del manejo del inglés de la población estudiantil. Posteriormente, se realizó un video explicativo en español sobre la actividad a realizar. 


\section{Las actividades}

Como primera actividad de mediación, se realizó un diagnóstico para determinar el grado de manejo del inglés de cada estudiante. Esta prueba se realizó al inicio del cuatrimestre, con el fin de recopilar datos útiles para el diseño de las estrategias indicadas para la mediación del material. Sobre todo, porque en nuestro país no todos reciben inglés desde la enseñanza primaria; de hecho, se dan casos en los que los estudiantes tienen su primer contacto con el inglés durante la universidad.

En segundo lugar, se realizó un video en español, mediante el cual se incentivó al estudiante para que estuviese receptivo a la introducción y explicación de la lectura correspondiente. Además, a través de este video se les ofrecieron recomendaciones básicas acerca de cómo realizar la lectura en inglés; por ejemplo, se les aconsejó marcar las palabras desconocidas y buscarlas en el diccionario antes de continuar con la lectura.

En tercer lugar, se planificó e incluyó el video en la plataforma, el cual consistió en la realización de un foro académico para mediar la lectura. Dicha actividad se implementó en la antepenúltima unidad académica de la materia; es decir, al final del primer cuatrimestre del 2021. Esto aseguró que cada estudiante ya hubiera visto y comprendido el $75 \%$ de los contenidos planteados en la asignatura, convirtiendo al material de apoyo (en inglés) en un complemento para ilustrar y enriquecer el aprendizaje adquirido durante el cuatrimestre. Esto, a su vez, facilitó una mejor compresión del contenido del material en inglés.

Cada foro académico contó con un video en español donde se explica la actividad y un material denominado: Mediación Pedagógica para el Artículo en Inglés. Allí se detallaron, en ambas lenguas, las instrucciones para realizar la actividad y utilizar los materiales en inglés.

Las actividades se diseñaron bajo las siguientes especificaciones:

1. Deben ser cortas, flexibles y dinámicas, de modo que no amenacen al discente.

2. Han de estar de acuerdo con las habilidades de la población estudiantil.

3. Las actividades deben plantearse en términos de falso y verdadero.

\section{DISCUSIÓN DE RESULTADOS}

La cantidad de matrícula global en las tres asignaturas fue de 653 estudiantes. Sin embargo, las y los participantes fueron 255 , es decir, un $40 \%$ de la población estudiantil matriculada. Participar en la actividad no fue obligatorio ni le fue asignado un valor porcentual de la nota. A partir de los hallazgos, se recomienda que los materiales utilizados durante la práctica pedagógica sean diseñados bajo criterios muy específicos, detallados a continuación:

Características de forma para la lectura:

- Deben ser cortas. La extensión de las lecturas no debe superar las 5 páginas.

- Deben enriquecerse con imágenes que expliquen el contenido.

- El formato del artículo debe ser amigable con el lector; es decir, presentarse en letra grande y con los márgenes recomendados.

Características de forma para los videos:

- Debe ser corto. No debe superar los 4 minutos de duración.

- Debe tener sonido y texto en inglés. 
- Se recomienda realizar una síntesis del contenido y luego una sesión de preguntas conducentes a la reflexión.

Características de fondo para la lectura.

- Se recomienda que el contenido del artículo complemente y refuerce el conocimiento del estudiante.

- Que el vocabulario utilizado sea simple y de fácil comprensión; además, debe evitarse el empleo de vocabulario técnico.

Características de fondo para los videos.

- Se recomienda que el contenido del video complemente y refuerce el conocimiento del estudiante.

- Que el vocabulario utilizado sea simple y de fácil comprensión, además, debe evitarse el empleo de vocabulario técnico.

Por otro lado, de los 255 participantes del estudio, el $10 \%$ sostiene no conocer del todo el inglés, lo que representa un porcentaje muy alto. Un $48,6 \%$ afirma ubicarse en un nivel básico, el 18,4\% se sitúa en un nivel intermedio. Solo el $0,7 \%$ asegura situarse en un nivel avanzado. Estos resultados se representan gráficamente por asignatura en la figura 2.

Figura 2. Percepción del nivel de inglés de las personas estudiantes participantes en la investigación.

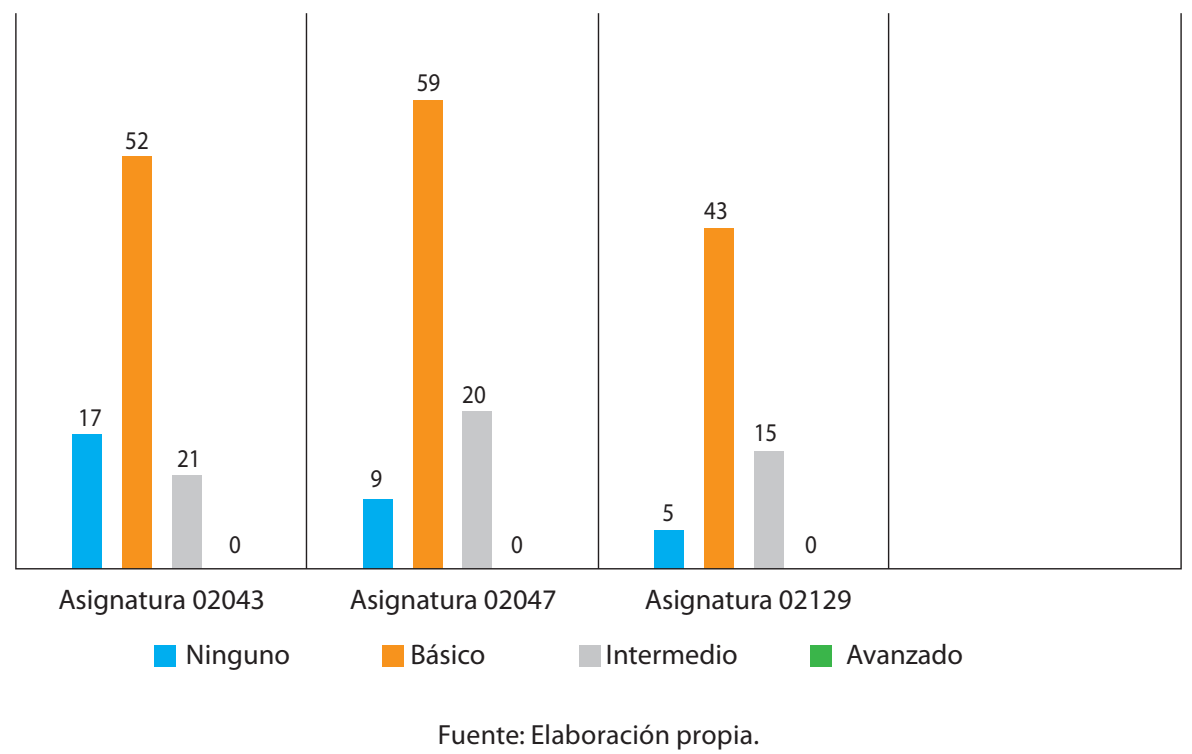

La participación del estudiantado en el foro de la lectura en inglés como material de apoyo, presenta los siguientes resultados, tal como se ilustra en la figura 3: la asignatura Material Didáctico para Preescolar, código 02043, tuvo una matrícula general de 187 estudiantes, de los cuales 81 participaron en el foro, para un $43 \%$ de participación. En la asignatura Introducción a la Educación Preescolar, código 02047, la matrícula fue de 256 estudiantes para un $37 \%$ de participación, es decir, 95 estudiantes participaron en el foro. Por último, en la asignatura Actividad Lúdica para Preescolar, código 02129, la matrícula fue de 212 estudiantes para un $37 \%$ de participación. Los datos indican que la actividad tuvo una participación aceptable, a pesar de no tener carácter obligatorio. 
Figura 3. Participación del estudiantado por asignatura en el foro de inglés.

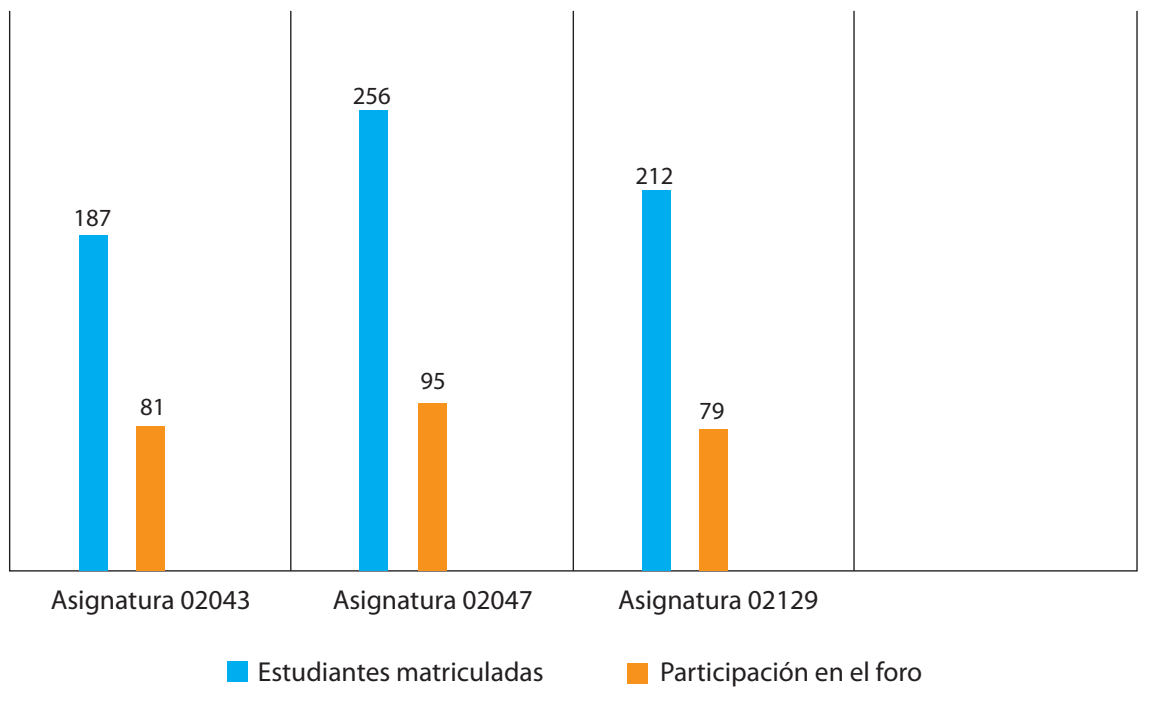

Fuente: Elaboración propia.

Quienes participaron en esta investigación perciben de forma distinta las lecturas en inglés escogidas para este estudio; sin embargo, la mayoría coincide en que fueron de fácil comprensión, hecho representado gráficamente en la figura 4.

Figura 4. Percepción del estudiantado sobre la comprensión de la lectura

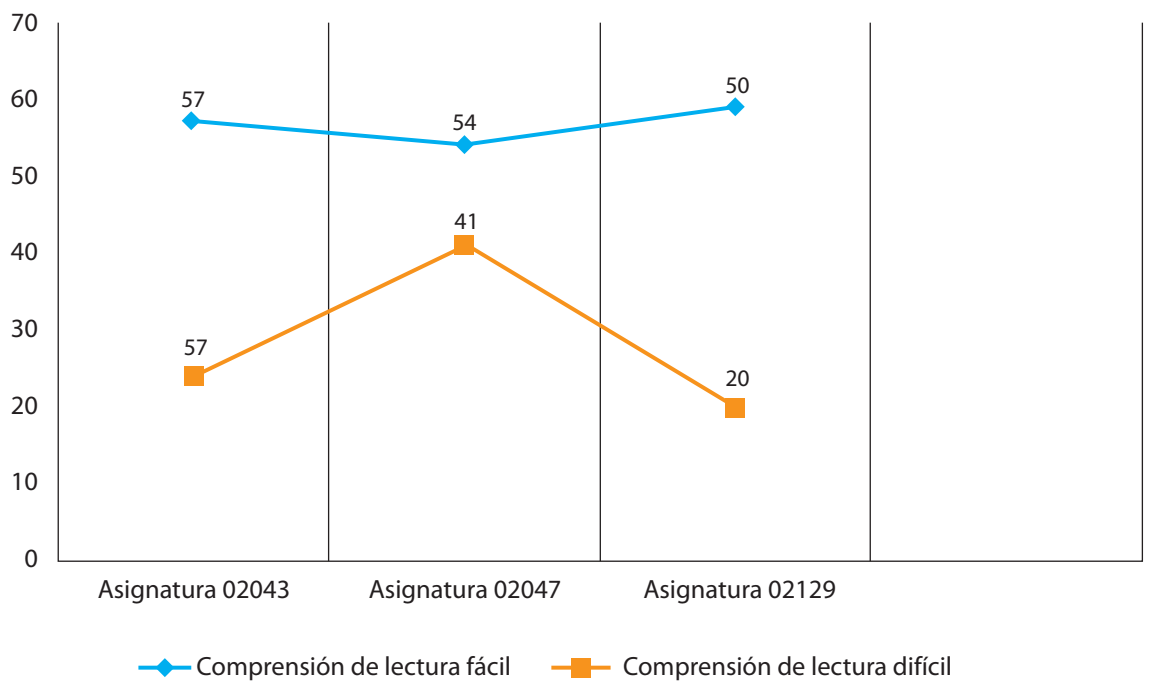

Fuente: Elaboración propia.

Para la asignatura Material Didáctico para Preescolar, código 02043, se escogió el artículo denominado: Teacher Inquiry on the Influence of Materials on Children's Learning, donde se aborda la temática sobre la influencia de los materiales y la persona docente en el aprendizaje de los infantes a su cargo. En esta asignatura, para el $57 \%$ la lectura fue de fácil comprensión.

En el caso de la asignatura Introducción a la Educación Preescolar, código 02047, se eligió el artículo Helping others understand academic rigor in teachers' developmentally appropriate practices, el cual 
aborda el rigor académico por parte de la persona docente para adquirir prácticas apropiadas. En esta asignatura el $54 \%$ percibió la lectura como de fácil comprensión.

Por último en la asignatura Actividad Lúdica para Preescolar, código 02129, se seleccionó el artículo: Teaching-interactive method used in the teaching-learning process, donde se trata el método interactivo para el aprendizaje. En esta asignatura para el $43 \%$ la lectura fue de fácil comprensión.

Los tres artículos fueron seleccionados con miras a que el estudiante se enriquezca con las diversas temáticas estudiadas durante el cuatrimestre, aumentando así su acervo de conocimiento. Por tal razón se subieron a la plataforma en la antepenúltima unidad académica del cuatrimestre. En general, para la mayoría de los estudiantes la lectura fue de fácil comprensión.

Por otro lado, la mediación pedagógica se realizó desde el aula virtual de cada asignatura. Las estrategias elegidas fueron: la basada en tareas y el aprendizaje cooperativo. A partir de estas estrategias se optó por un ítem de predicción, en este caso con preguntas de falso y verdadero, con el fin de que la población estudiantil utilizara los conocimientos previos, la información que tenían en su memoria, el contexto, las ilustraciones, el título, o cualquier otra información fácil de responder y de predecir las oraciones planteadas en la actividad. Cabe destacar que las instrucciones y el ejercicio de falso y verdadero se le presentaron primero en inglés y después se les tradujo al español.

De la misma manera se hizo en la asignatura Material Didáctico para Preescolar, código 02043. Se plantearon seis oraciones para ser contestadas con un falso o verdadero, basándose en la lectura y los conocimientos previos. Ejemplo del ejercicio:

Instructions: According to the article, Teacher Inquiry on the Influence of Materials on Children's Learning, decide if the statement is true $(\mathrm{T})$ or false $(\mathrm{F})$.

1. Piaget proposed that children learn by just creating their own theories ( ).

2. Vygotsky stressed the importance of internal process because it helps solving a conflict and creating an object ( ).

La lectura de la asignatura Introducción a la Educación Preescolar, código 02047 permitió plantear una pregunta más. En este caso se establecieron 7 afirmaciones para que cada estudiante contestara si eran falsas o verdaderas. Ejemplo del ejercicio:

Instructions: According to the article, Helping others understand academic rigor in teachers' developmentally appropriate practices, decide if the statement is true $(T)$ or false $(F)$.

1. According to the article, teachers should consider academic rigor as an indicator of quality ( ).

2. The author mentions an example in which she practices morning routines through free activities and no organization ( ).

En la asignatura Actividad Lúdica, código 02129, se plantearon siete oraciones para que la y el estudiante contestaran si eran falsas o verdaderas. Ejemplo del ejercicio:

Instructions: According to the article, Teaching interactive method used in the teaching learning process, decide if the statement is true $(\mathrm{T})$ or false $(\mathrm{F})$.

1. The word game means: the action to play ( ).

2. The game is not an important part of education ( ).

Como se observa en la figura 5, la percepción de los estudiantes en cuanto a la dificultad de los ítems de falso y verdadero es positiva. En la asignatura Material Didáctico para Preescolar, código 02043, más 
del $50 \%$ afirma que la materia presenta poca dificultad. En el caso de la asignatura Introducción a la Educación Preescolar, código 02047, para la mayoría de los participantes la asignatura ofrece un alto grado de dificultad, mientras que para Actividad Lúdica, código 02129, ocurre lo contrario, es decir, la considera de un nivel bajo de dificultad.

Figura 5. Percepción del estudiantado sobre la dificultad de la actividad de falso y verdadero.

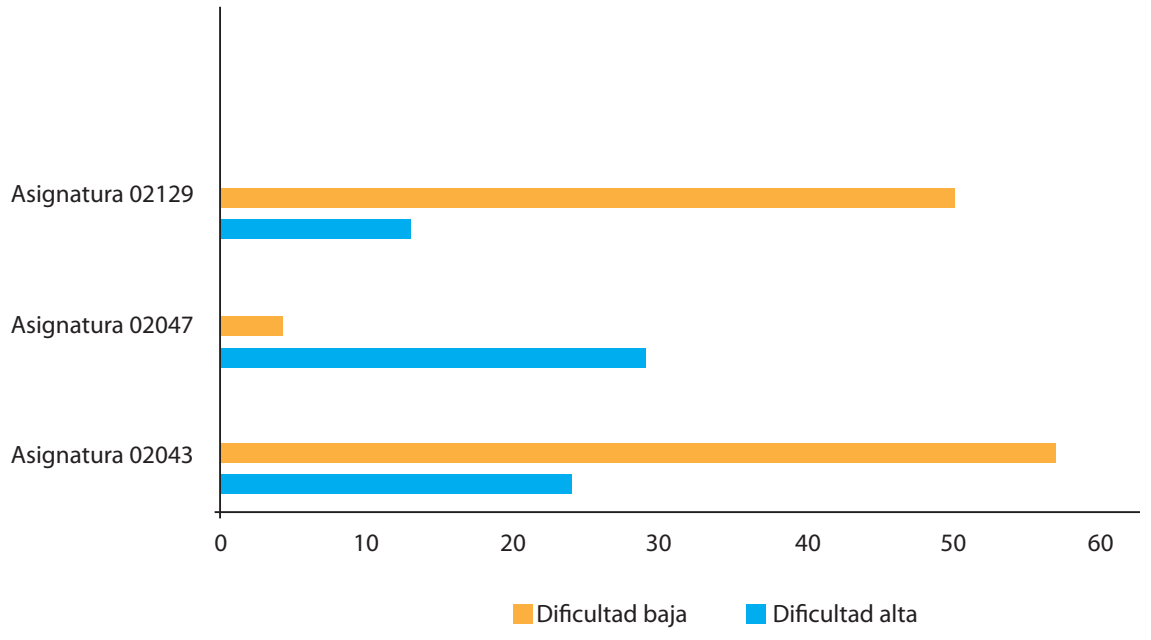

Fuente: Elaboración propia.

Otro resultado importante de socializar es el ítem de falso y verdadero, ya que más del $60 \%$ del estudiantado participante de la actividad contestó correctamente todas las oraciones y el $40 \%$ restante tuvo al menos una respuesta incorrecta.

\section{Discusión}

Este artículo es un aporte a la literatura relacionada con la mediación pedagógica, específicamente para la mediación con materiales de apoyo en otro idioma, en este caso el idioma inglés para una población universitaria con un dominio básico del mismo. Contrariamente a las expectativas negativas que se tenían sobre esta mediación antes de iniciar el estudio, este demostró que si es posible utilizar materiales de apoyo en otro idioma aunque la población estudiantil posea un dominio básico del mismo. La utilización de artículos y videos en inglés en las y los estudiantes universitarios del nivel de diplomando parecía resultar una tarea imposible y difícil, sin embargo, este trabajo nos demuestra que si es posible utilizar materiales en otro idioma, aunque la población estudiantil no posea un dominio de este. Por otro lado, resalta la relevancia de las estrategias metodológicas que realice la persona docente encargada de la asignatura para que la mediación sea posible.

Las estrategias metodológicas para mediar el aprendizaje en otro idioma, como afirma Rodríguez (2014), deben romper con una serie de limitaciones que acompañan a la persona estudiante desde la infancia y que no le permiten un acercamiento y aprendizaje de otro idioma en la vida adulta. Por esta razón, las estrategias basadas en tareas y trabajo colaborativo se convirtieron en una herramienta fundamental para el éxito de la mediación de los materiales elegidos, ya que les permitió a las y los estudiantes dejar de lado los temores y limitaciones en otro idioma para utilizar todos los recursos, los materiales, las vivencias y los aprendizajes encontrados hasta el momento para resolver el ejercicio de predicción subido en la plataforma. La actividad de predicción, en este caso el ítem de falso y verdadero, como lo afirma la Agencia Colombiana de la Calidad de la Educación (2019), le permitió al estudiantado utilizar 
los conocimientos previos, la memoria, el contexto, las ilustraciones, el título o cualquier otra información para responder y predecir la respuesta a lo que le solicitaron, sin que el nivel del idioma fuera un problema.

No obstante, otro aspecto fundamental para el éxito en la mediación fue la elección del material didáctico a utilizar y el periodo del cuatrimestre en el que se debe realizar. En este caso se decidió utilizar la antepenúltima unidad temática, momento cuando la población estudiantil ya habría adquirido los conocimientos básicos de los temas propios de cada asignatura, facilitando la comprensión del artículo y la capacidad de asociar el conceptos asimilados con la lectura. Además, se optó por trabajar con lecturas y videos.

Es indispensable que el material elegido tenga un lenguaje claro y simple; por otra parte, el artículo no debe ser muy extenso, debe contener imágenes alusivas al tema, subtítulos y frases sobresalientes para facilitar la identificación de las ideas principales de la lectura. En Los videos deben estar subtitulados en inglés y al finalizar conviene realizar un pequeño resumen de su contenido.

Otro hallazgo de este estudio se refiere a que la carga académica de la asignatura no afecta la participación si el incentivo es mayor y la mediación es la correcta. Esto quedó en evidencia pues la actividad no fue obligatoria ni fue calificada y aun así la participación fue de un $40 \%$ de las y los estudiantes matriculados. Se incentivó a cada estudiante a participar activamente y en muchos casos a que realizara el primer acercamiento hacia un segundo idioma.

El aprendizaje es un proceso auto organizativo y constante, que implica una transformación de las experiencias de la vida, que se aprende a través de todos los sentidos mediante un proceso corporal relacionado con el placer, el disfrute. Por lo tanto, como seres humanos se es un compendio de experiencias formales y vivenciales, surgido de procesos espontáneos y emergentes, sin propósitos intencionales o conscientes (Assmann, 2002). Esto permitió entender la mediación como una oportunidad para conducir al estudiante a una reflexión acerca de la importancia de dominar un segundo idioma en el quehacer docente, en la formación continua y en las oportunidades laborales.

\section{SIINTESIS Y REFLEXIONES FINALES}

El estudio sobre la mediación pedagógica del inglés para estudiantes, cuyo manejo del idioma es básico, abordó las siguientes interrogantes: ¿cuáles estrategias de mediación requiere el estudiantado para atender material didáctico en inglés sin tener el dominio óptimo?, ¿es el nivel del idioma de los materiales seleccionados un aspecto fundamental a considerar?, ¿cuál periodo (al inicio, al intermedio o al final) del cuatrimestre es el momento propicio para realizar las actividades?

Se determinó que las estrategias de trabajo en tareas y trabajo colaborativo son apropiadas para mediar materiales didácticos en una población sin dominio del idioma, pues estas no se centran en el idioma propiamente ni la lengua es el objeto de estudio, pues se basan en la vida real y en cómo permiten realizar tareas relacionadas con el bagaje de conocimiento previo de cada estudiante; por tal razón, los ítems de predicción constituyen la mejor actividad para mediar los materiales, pues se comprobó que en dicho tipo de ejercicio cada estudiante utilizó todos los recursos y conocimientos previos a su disposición para responder el ejercicio.

En segundo lugar, la elección de los materiales resulta fundamental para realizar con éxito su mediación, por lo que deben cumplir con ciertas características de fondo y forma para propiciar la incentivación extrínseca e intrínseca de la persona estudiante, con miras a que participe y resuelva las actividades propuestas. 
Dentro de las principales características de los materiales pueden citarse: un lenguaje simple no técnico, una extensión no mayor a las cinco páginas, poseer imágenes alusivas al tema, poseer frases y subtítulos que faciliten el reconocimiento de las ideas fundamentales del artículo.

En tercer lugar, se destaca la importancia del cómo y cuándo presentar el material y realizar la actividad. En este aspecto fue posible identificar como el momento idóneo para incorporar el material de apoyo en otro idioma, aquel cuando la población estudiantil ha adquirido alrededor de un $75 \%$ de los contenidos de la asignatura. Esto le permitirá empoderarse sobre su proceso de aprendizaje, asociar los conocimientos con la lectura en otro idioma y conseguir una mejor comprensión del tema del artículo.

\section{REFERENCIAS}

Agencia Colombiana de la Calidad de Educación. (2019). Estrategia de comprensión lectora hacer predicciones. Recuperado de http://archivos.agenciaeducacion.cl/ACE_Estrategia_Hacer_predicciones.pdf

Assmann, H. (2002). Placer y Ternura en la Educación: Hacia una Sociedad Aprendiente. Madrid: Narcea S. A.

Barrantes, R. (2014). Investigación, Un camino al conocimiento, Un Enfoque Cualitativo, Cuantitativo y Mixto. San José, Costa Rica, Editorial EUNED.

Barquero, K. (2019). Mayoría de docentes de inglés en la región con dominio básico, advierten estudio. LaRepublica.Net https;//www.larepublica.net/noticia/mayoría-de-docentes-de-ingles-de-la-regioncon-dominio-basico-advierte-estudio

Bolívar, A. (2017). Docencia e investigación en la universidad: de una relación problemática a una productiva. Revista de Gestión de la Innovación en Educación Superior REGIES, 2, p.p.11-33. Issn 0719742X.; E-Isnn: 0719-7624

Cerdas, D. (2016, 1 diciembre). Solo una cuarta parte de los costarricenses habla inglés. La Nación. Recuperado de https://www.nacion.com/el-pais/educacion/solo-una-cuarta-parte-de-loscostarricenses-habla-ingles/UU726UVRWFFOHPTDTIY53FUUAE/story/

Chick, Nancy. (s.f.). A scholarly approach to teaching. In Scholarship of teaching and learning: A guide from the Vanderbilt University Center for Teaching. Retrieved from https://my.vanderbilt.edu/ sotl/understanding-sotl/a-scholarly-approach-to-teaching/.

Chocarro, E.; Sobrino, A \& González, M. (2013). Scholarship of Teaching and Learning: Un modelo de desarrollo profesional de los profesores universitarios. Revista Electrónica Interuniversitaria de Formación del Profesorado, 16(1), 5--14. DOI: http://dx.doi.org/10.6018/reifop.16.1.179401

EF-EPI. (2019). El ranking mundial más grande según su dominio del inglés. Ef. Com. https://www.ef.com/cl/epi/

García, J. (2016). La enseñanza de la segunda lengua en el aula de Infantil a través de las TIC: propuesta didáctica. (Tesis de Licenciatura) Universidad de Valladolid, España. Recuperado de: https://uvadoc.uva.es/bitstream/10324/18548/1/TFG-O\%20749.pdf

Garza, J. (2019). Costa Rica tiene el segundo mejor inglés no nativo de Latinoamérica. LaRepública.Net. https://www.larepublica.net/noticia/costa-rica-tiene-el-segundo-mejor-ingles-no-nativo-delatinoamerica

González, W. y Garbanzo, P. (2016). Implementación de un programa educativo en Artes Plásticas basado en el Aprendizaje Cooperativo sobre la motivación en estudiantes de secundaria. (Tesis de Licenciatura) Universidad Nacional, Costa Rica. 
Rodríguez, N. (2014). Creencias y representaciones de los profesores de lenguas extranjeras sobre la influencia de los factores motivacionales y emocionales en los alumnos y en las alumnas. Recuperado de http://www.ugr.es/ portalin/articulos/PL_numero21/12\%20\%20Nieves.pdf

Morales, S. (2008). La efectividad de un modelo de aprendizaje combinado para la enseñanza del inglés como lengua extranjera: estudio empírico. Revista de Lingüística Teórica y Aplicada. 95-118. CL ISSN 0033 - 698X

Sabokrouh, F. (2014). The Effect of EFL Teachers' Attitude toward English Language and English Language Proficiency on Their Sense of Efficacy. Vol. 7, No. 1; ISSN 1916-4742 E-ISSN 1916-4750

Rafieyan, V; Orang, M; Bijami, M; Sharafi, M; Nejad \& Lin Siew Eng. (2014). Language Learners' Acculturation Attitudes. English Language Teaching; Vol. 7, No. 1; ISSN 1916-4742 E-ISSN 1916-4750 